\title{
Analysis of the Interaction of Human Neuroblastoma Cell-Derived Cytochalasin B Induced Membrane Vesicles with Mesenchymal Stem Cells Using Imaging Flow Cytometry
}

\author{
Valeriya V. Solovyeva $^{1} \cdot$ Kristina V. Kitaeva $^{1}$ - Daria S. Chulpanova ${ }^{1} \cdot$ Svetlana S. Arkhipova $^{1} \cdot$ Ivan Yu. Filin $^{1}$. \\ Albert A. Rizvanov' ${ }^{1}$
}

Accepted: 29 November 2021 / Published online: 4 March 2022

(c) The Author(s), under exclusive licence to Springer Science+Business Media, LLC, part of Springer Nature 2022

\begin{abstract}
At present, there is an increasing interest in the potential role of extracellular vesicles (EVs), acting as multi-signal messengers of the tumor stroma, in the development and progression of tumor. Tumor cell-derived EVs are considered a potential vector for the targeted delivery of antitumor agents due to the ability to fuse with parental cells through endocytosis and release their contents into the cytoplasm of the recipient cell. Tumor cell-derived EVs could be also used for priming immune cells and therapeutic vaccine development. It is also known that mesenchymal stem cells (MSCs) have a tropism toward tumor niches. It is believed that MSC migration to the tumor is due to its inflammatory signaling. Presumably, with the accumulation of MSCs at tumor sites, these cells differentiate into pericytes or tumor-associated fibroblasts, thereby forming a supporting tumor growth microenvironment. However, besides the ability to promote tumor progression, MSCs can also suppress its growth by inhibiting proliferation and cell cycle progression, and angiogenesis. Thus, the further studies of the MSC role in TME and MSC interaction with other cells of the tumor stroma, including through EVs, are of particular interest. To increase the yield of vesicles the isolation method based on pharmacological disorganization of the actin cytoskeleton induced by treating with cytochalasin B was used in this study. In this investigation the interaction of SH-SY5Y neuroblastoma cell-derived membrane vesicles, obtained using cytochalasin B (CIMVs), with human bone marrow-derived MSCs was analyzed using imaging flow cytometry. Using transmission electron microscopy, it was shown that CIMVs have a size similar to that of natural microvesicles, which is $100-1000 \mathrm{~nm}$. Using imaging flow cytometry, it was shown that after $24 \mathrm{~h}$ of co-cultivation $6 \%$ of the MSCs contained a large number of CIMVs, and $42 \%$ of the MSCs contained a small amount of CIMVs. Cultivation of MSCs with SH-SY5Y cell-derived CIMVs also induced dose-dependent decrease in the expression of CD markers typical for MSCs. Thus, the internalization of SH-SY5Y cell-derived CIMVs within MSCs and the ability of the CIMVs to modulate immunophenotype of the recipient cells were shown. However, further studies are required to determine the effect of CIMVs on pro- or antioncogenic phenotype and function of MSCs.
\end{abstract}

Keywords Extracellular vesicles $\cdot$ Exosomes $\cdot$ Membrane vesicles $\cdot$ Mesenchymal stem cells $\cdot$ Neuroblastoma cells · Cytochalasin B · Imaging flow cytometry

\section{Introduction}

Due to the key role in intercellular communication, considerable interest is directed to the study of extracellular vesicles (EVs), membrane vesicles secreted into the extracellular environment by almost all cell types of the body.

Albert A. Rizvanov

rizvanov@gmail.com

1 Kazan Federal University, Kazan, Russia
Depending on the cellular origin, EVs can be classified into exosomes, microvesicles (MVs) and apoptotic bodies (ABs) [1]. Exosomes are formed by endocytosis. MVs are released by direct budding and fusion of the plasma membrane [2]. Both exosomes and MVs carry the cargo of the parental cell represented by various proteins and nucleic acids. Unlike other types of EVs, ABs are formed only when cells are fragmented during apoptosis [1].

At present, there is an increasing interest in exploring the potential role of EVs in tumor development and progression. Besides tumor cells, the tumor microenvironment (TME) 
contains a number of other cell types, such as fibroblasts, epithelial and endothelial cells, lymphocytes, adipocytes, inflammatory cells, and mesenchymal stem/stromal cells (MSCs) [3]. EVs act as key mediators in maintaining and spreading the tumor by mediating intercellular communication in TME and thus inducing phenotypic modifications of the recipient cells [4]. Tumor cells, among other ways, use EV-mediated intercellular signaling to maintain tumor vascularization, invasion, and immune escape, as well as to form an aggressive phenotype and drug resistance [1]. EVs can carry oncogenes and onco-miRNAs, thereby modulating the activity of various signaling pathways in recipient cells and playing a crucial role in tumor growth and metastasis [5].

MSCs if released to TME can be differentiated into myofibroblasts [6], tumor-associated fibroblasts (TAMs) [7], or pericytes, thus supporting tumor growth [8], while also MSCs can induce epithelial-mesenchymal transition (EMT), a process in which epithelial cells lose their polarity and adhesiveness and acquire migratory and invasive properties [9]. Pro-tumor properties of MSCs are also due to the secretion of various trophic factors and cytokines, which have a favorable effect on the survival of tumor cells (stromal cell-derived factor 1 (SDF-1), hepatocyte growth factor (HGF), insulin-like growth factor 1 (IGF-1)), their proliferation (epidermal growth factor (EGF), HGF, nerve growth factor (NGF), transforming growth factor $\alpha(\mathrm{TGF}-\alpha)$ ), and tumor vascularization (vascular endothelial growth factor (VEGF), SDF-1) [10]. MSCs can inhibit the anti-tumor immune response through either TAMs or bone marrow stromal cells [11]. However, MSCs have been also reported to exhibit antitumor properties in TME, which are due to the suppression of the phosphoinositide-3 kinase/protein kinase $\mathrm{B}$ signaling pathway (PI3K/Akt). Such a pro-apoptotic effect of MSCs has been described in hepatocellular carcinoma, glioma, and Kaposi's sarcoma [12], as well as prostate and breast cancer [13]. Other possible mechanisms that contribute to the antitumor potential of MSCs include apoptosis mediated by the activation of TNF-related apoptosis-inducing ligand (TRAIL), G1 cell cycle arrest, and expression of oncosuppressor genes [14]. MSCs that show a strong tropism to tumor niches can be derived from bone marrow, adipose tissue, and also the umbilical cord [15]. MSCs are known to migrate in multiple inflammatory microenvironments in multiple tumor or wound models [16] and irradiated environments [17]. Understanding the role of MSCs in TME is important for developing new therapeutic strategies for MSC-based targeted delivery of anticancer agents toward tumor niches [14, 18, 19].

Thus, the further studies of the MSC role in TME and MSC interaction with other cells of the tumor stroma, including through EVs, are of particular interest. In order to increase the yield of obtained vesicles in this work, the isolation method based on pharmacological disorganization of the actin cytoskeleton by treating the cells with cytochalasin B and vortexing was used. Cytochalasin B allows obtaining nucleus-free vesicles, surrounded by a cytoplasmic membrane and containing the content of parental cell cytoplasm. A distinctive feature of cytochalasin B-induced membrane vesicles (CIMVs) is the lack of active mechanism of cargo sorting during CIMV generation [20].

This paper examines the interaction of CIMVs isolated from tumor neuroblastoma cells with bone marrow-derived MSCs. For the first time, CIMVs obtained from neuroblastoma SH-SY5Y cells were characterized using transmission electron microscopy and flow cytometry, as well as the interaction of CIMVs from tumor neuroblastoma cells with MSCs was assessed using imaging flow cytometry. Also, the influence of tumor cell-derived CIMVs on the immunophenotype of bone marrow-derived MSCs was investigated.

\section{Materials and Methods}

\subsection{Cells and Culture Conditions}

Bone marrow samples were provided by the Republican Clinical Hospital for research purposes in accordance with ethical standards and current legislation (the protocol was approved by the Committee on Biomedical Ethics of Kazan Federal University (No. 3, 03/23/2017)). Written informed consent was obtained from donors. MSCs from human bone marrow were isolated in a ficoll density gradient $(1.077 \mathrm{~g} /$ $\mathrm{cm}^{3}$, PanEco, Russia), followed by adhesion of cells to the surface of culture plastic as previously described [21]. SHSY5Y human neuroblastoma cells were obtained from the American Type Culture Collection (ATCC number: CRL2266, Manassas, VA, USA). MSCs and SH-SY5Y were cultured in DMEM/F12 (PanEco, Russia) supplemented with $10 \%$ fetal bovine serum (FBS) (Invitrogen, USA), $2 \mathrm{mM}$ L-glutamine (PanEco, Russia), penicillin (100 U/ml), and streptomycin $(100 \mu \mathrm{g} / \mathrm{ml})$ (Biolot, Russia). Cells were maintained at $37{ }^{\circ} \mathrm{C}$ in a humid atmosphere with a content of $5 \% \mathrm{CO}_{2}$. Sub-confluent cells (approximately $80 \%$ confluent) were passaged using $0.25 \%$ trypsin-EDTA solution (Invitrogen, USA).

\subsection{Immunophenotyping}

MSC immunophenotyping was performed using the antibodies to CD29 (\#87,106, Sony, USA), CD44 (\#51-9,007,656, BD Biosciences, USA), CD73 (\#51-9,007,649, BD Biosciences, USA), CD90 (\#51-9,007,657, BD Biosciences, USA), and CD105 (\#323,218, BioLegend, USA); a cocktail of PE-conjugated antibodies hMSC Negative Cocktail from the Human MSC Analysis Kit (\#562,245, BD Biosciences, 
USA) was used as a negative control to identify potential contaminants with hematopoietic cells [22,23]. The staining results were analyzed using FACS Aria III (BD Biosciences, USA) and BD FACSDiva ${ }^{\mathrm{TM}}$ software version 7.0.

\subsection{CIMV Production}

CIMVs were isolated as previously described [24]. SHSY5Y cells were washed twice with Dulbecco's PhosphateBuffered Saline (DPBS, PanEco, Russia) and incubated in DMEM/F12 without serum containing $10 \mu \mathrm{g} / \mathrm{ml}$ of cytochalasin B (Sigma-Aldrich, USA) for $30 \mathrm{~min}\left(37^{\circ} \mathrm{C}, 5 \% \mathrm{CO}_{2}\right)$. At the end of incubation, the cell suspension was vortexed vigorously for $30 \mathrm{~s}$ and centrifugated (500 $\mathrm{g}$ for $10 \mathrm{~min}$ ). The supernatant was subjected to two subsequent centrifugation steps (700 $g$ for $10 \mathrm{~min}$ and $12000 \mathrm{~g}$ for $15 \mathrm{~min}$ ). The resulting pellet contained CIMVs from SH-SY5Y cells. Total protein concentration in CIMV samples was determined using the Pierce ${ }^{\mathrm{TM}}$ BCA Protein Assay Kit (Thermo Fisher Scientific, USA) according to manufacturer's recommendations. The results were measured at wavelength $562 \mathrm{~nm}$.

To determine the size of the isolated CIMVs, flow cytometry analysis was performed using SPHERO ${ }^{\mathrm{TM}}$ Nano Fluorescent Particle Size Standard Kit (Spherotech, USA).

\subsection{Analysis of CD Marker Expression in the CIMVs}

CIMVs were isolated from $1 \times 10^{6} \mathrm{SH}-\mathrm{SY} 5 \mathrm{Y}$ cells, washed once with PBS, and stained with $1 \mu \mathrm{l}$ of anti-CD63 (PerCP/ Cy5.5) (\#353,020, BioLegend, USA), anti-CD81 (PE/Cy7) (\#349,512, BioLegend, USA), and anti-tumor susceptibility 101 (TSG101) (PE) (\#ab209927, Abcam, UK) antibodies against specific surface markers for $30 \mathrm{~min}$ in the dark at room temperature (RT). After that, CIMVs were washed twice with PBS and analyzed using flow cytometer FACS Aria III (BD Biosciences, USA) and BD FACSDiva ${ }^{\mathrm{TM}}$ software version 7.0. To analyze the presence of intracellular markers heat shock protein $70 \mathrm{kDa}(\mathrm{Hsp} 70)$ and calnexin, newly isolated CIMVs were fixed with $0.01 \%$ formaldehyde for $15 \mathrm{~min}$ at RT and washed with PBS for $5 \mathrm{~min}$. Next CIMV membranes were permeabilized using $0.1 \%$ Triton X-100 for $15 \mathrm{~min}$ at RT. After that, the samples were washed twice with PBS and stained with anti-Hsp70 (FITC) (\#648,004, BioLegend, USA) and anti-Calnexin (Alexa Fluor 594) (\#ab203439, Abcam, UK) antibodies for $30 \mathrm{~min}$ in the dark at RT. Stained vesicles were washed twice with PBS and analyzed using FACS Aria III (BD Biosciences, USA) and BD FACSDiva ${ }^{\mathrm{TM}}$ software version 7.0.

\subsection{Transmission Electron Microscopy}

CIMVs were fixed in $2.5 \%$ glutaraldehyde on PBS solution $\left(12 \mathrm{~h}\right.$ at $\left.4{ }^{\circ} \mathrm{C}\right)$, and then washed twice with phosphate buffer by centrifugation, and precipitated vesicles were resuspended in $100 \mu \mathrm{l}$ of phosphate buffer. The resulting solution $(5 \mu \mathrm{l})$ was dropped on nickel grids coated with FormvarCarbon film (200 mesh, Sigma-Aldrich, USA), and the sample were contrasted with uranyl acetate and lead citrate. The grids were dried for $12 \mathrm{~h}$ in air at $37^{\circ} \mathrm{C}$. Samples were examined using transmission electron microscope HT7700 (Hitachi, Japan) (accelerating voltage: $100 \mathrm{kV}$ ) at the KFU Interdisciplinary Center for Analytical Microscopy.

\subsection{Cytoplasmic Membrane Staining}

Fluorescent cell membrane staining of MSCs and CIMVs was performed in a serum-free medium with Vybrant Cell Labeling Solutions kit (Thermo Fisher Scientific, USA) $[21,25]$ using DiO (green emission spectrum) and DiD (red emission spectrum) dyes, respectively, according to manufacturer's recommendations. A qualitative assessment of staining was performed on an Axio Observer.Z1 fluorescence microscope (Carl Zeiss, Germany) using AxioVision Rel. 4.8 software.

\subsection{Imaging Flow Cytometry}

After fluorescent staining, $1 \times 10^{6} \mathrm{MSCs}$ were seeded onto 6-well plate and cultured in full DMEM/F12 medium containing $100 \mu \mathrm{g} / \mathrm{ml}$ of CIMVs for $24 \mathrm{~h}$ at $37{ }^{\circ} \mathrm{C}$ in $5 \% \mathrm{CO}_{2}$. After that, cells were trypsinized and washed with DPBS three times by centrifugation (at $500 \mathrm{~g}$ for $5 \mathrm{~min}$ ). Cell pellets were resuspended in DPBS. Imaging flow cytometry was performed using the 5-laser ImageStreamX Mark II (AmnisEMD-Millipore, USA). Data were collected using Inspire software and further analyzed by Ideas software.

\section{Results and Discussion}

Currently, there is an increasing interest in the role of MSCs in tumor progression. One of the key factors in this process is the modulation of MSC properties through tumor cell-derived EVs. In this study, MSCs were isolated from human bone marrow. Immunophenotypic analysis showed that isolated MSCs had a mesenchymal stem cell phenotype and expressed surface markers CD29 (99.9\%), CD44 (100\%), CD73 (79.8\%), CD90 (99.5\%), and CD105 (91.3\%), while the expression of hematopoietic cell surface markers (CD11b, CD19, CD34, CD45, HLA-DR) was insignificant (Fig. 1).

There are several methods to isolate natural EVs from cell cultures. One of the most common methods is differential centrifugation, in which particles are separated based on their different densities or sizes. For additional purification, an extra step using sucrose or iodixanol cushion/gradient 

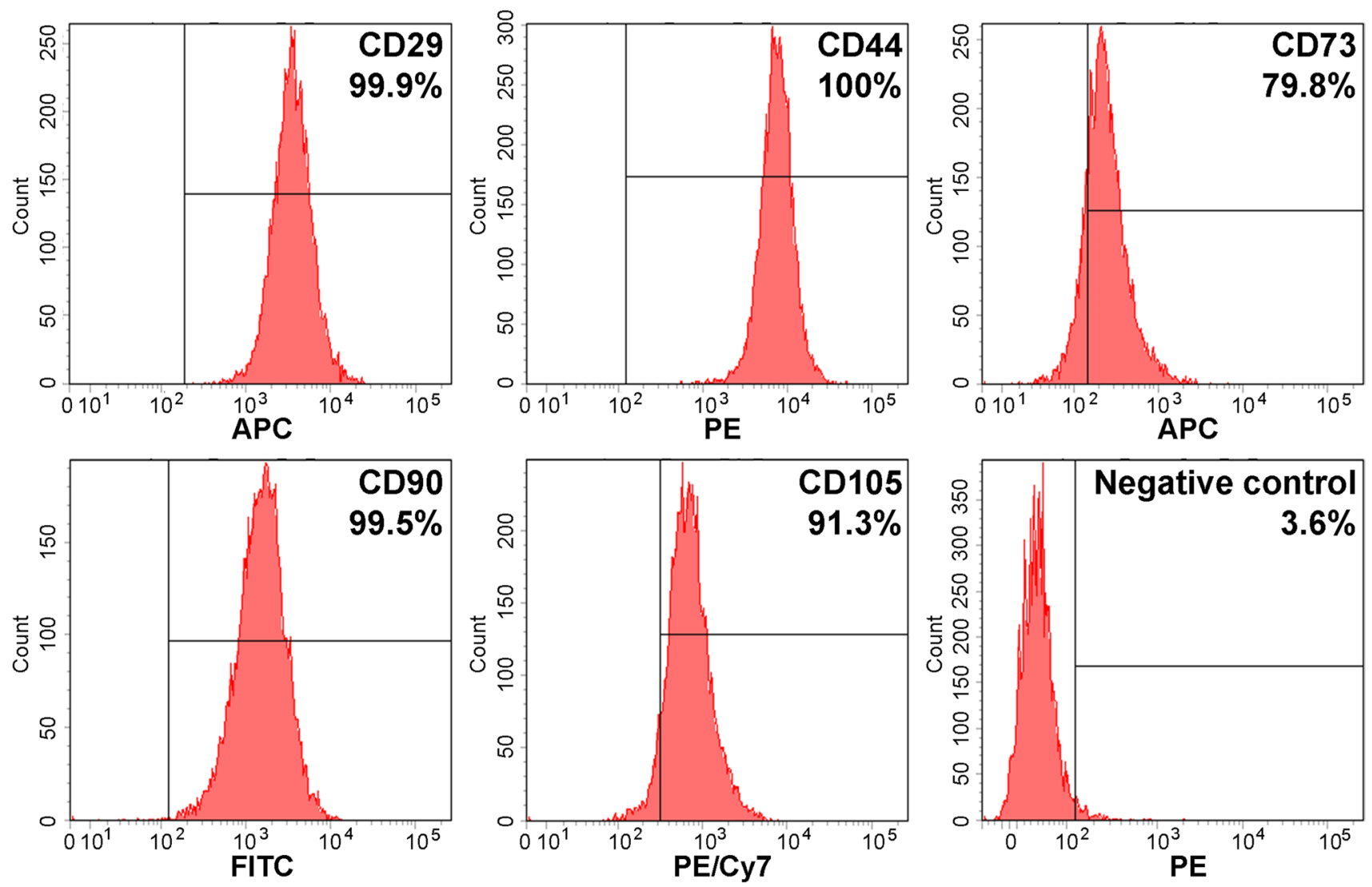

Fig. 1 Immunophenotypic characteristics of human bone marrow MSCs (passage 0). Negative markers, hematopoietic cell markers: CD34, CD11b, CD19, CD45, and HLA-DR

can be applied. Another common method for EV isolation is ultrafiltration, in which EVs are separated based on their size [26]. In addition to the abovementioned methods for the isolation of EVs polymer-based precipitation, size-exclusion chromatography and immunoaffinity are also used. Every approach of EV isolation has its advantages and disadvantages [27].

Neuroblastoma is the most common solid tumor in children, with the current survival rate of less than 50\% [28]. Tumor microenvironment cells, such as TAMs and MSCs, are presented in neuroblastoma tumors and contribute to tumor progression [29]. Therefore, the study of the effect of neuroblastoma tumor cells on the TME cells is important for understanding the contribution of tumor-stroma intercellular communication to tumor progression.

In the present work, to increase the yield of vesicles, human neuroblastoma cells SH-SY5Y were treated with cytochalasin B, a metabolite product of some mold fungi, resulting in cytokinesis inhibition. This compound causes the reorganization of cytoskeleton and induces the formation of EVs [30]. The use of cytochalasin B allows increasing the yield of membrane vesicles. Thus, the protein concentration of natural EVs harvested from $10^{8} \mathrm{MSC}$ was
$402.48 \pm 178.12 \mu \mathrm{g}$, whereas the protein concentration of CIMVs obtained from $10^{6}$ MSCs was about $1 \mathrm{mg}$ [29]. After SH-SY5Y cells were treated with cytochalasin B, CIMVs were obtained by active vortexing of the cell suspension followed by differential centrifugation. The obtained CIMVs were analyzed using TEM and flow cytometry. Resulted images (Fig. 2) show that CIMVs obtained from SH-SY5Y cells are 100 to $1000 \mathrm{~nm}$ in diameter and are round or irregular in shape. At the same time, 68\% CIMVs ranged in size from 220 to $450 \mathrm{~nm}$ and $27 \%$ CIMVs were from 450 to $880 \mathrm{~nm}$. The smallest number of particles $(2 \%$ and $2.6 \%$ ) was less than $220 \mathrm{~nm}$ and more than $880 \mathrm{~nm}$ in size, respectively. The fusion or the formation of conglomerates consisting of several CIMVs can be observed (Fig. 2). The obtained data allow to conclude that the size of SHSY5Y-derived CIMVs corresponds to the size of MVs $(100-1000 \mathrm{~nm})$ and exosomes $(40-100 \mathrm{~nm})$ obtained in natural way [1]. It is known that cytochalasin B-induced membrane vesicles retain cell surface receptors of parental cell, which can ensure their further interaction with target cells, and are capable of supporting the receptor-mediated signaling of the parental cell $[24,30,31]$. Most of the SHSY5Y-isolated CIMVs carried CD63 (54.2 $\pm 0.3 \%)$ and 
Fig. 2 Characteristics of SHSY5Y cell-derived CIMVs. A Transmission electron microscopy. B Flow cytometry analysis
A

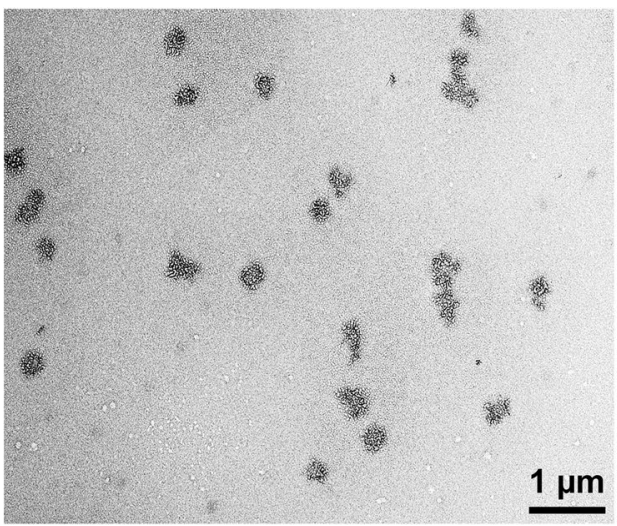

B

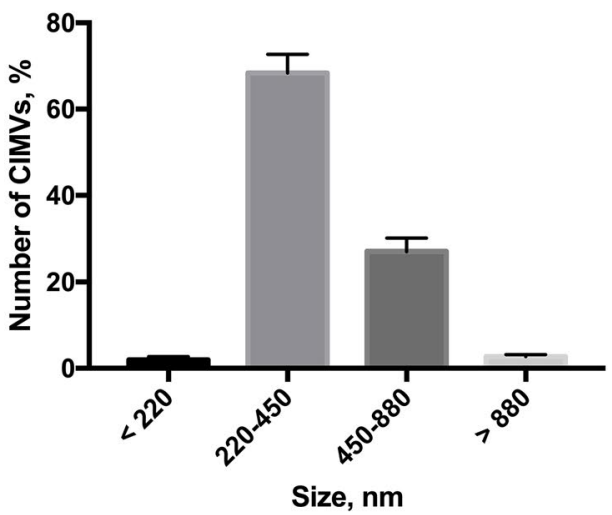

CD81 $(77.4 \pm 0.1 \%)$ on their surfaces. At the same time, the expression of TSG101 on the membrane of vesicles was limited $(6.6 \pm 0.1 \%)$. Presence of intracellular marker Hsp70 $(43.4 \pm 1.2 \%)$, but not calnexin $(0.7 \pm 0.2 \%)$, was also observed in the CIMVs (Fig. 3). CD63, CD81, and TSG101 are common markers of naturally produced EVs [32]. The presence of these markers on the surface of CIMVs indicates similarity of our vesicles and endogenous EVs. Hsp70 is cytosolic protein which is normally covered into vesicles during their formation [33]. We have also observed Hsp70 inside the CIMVs. At the same time, calnexin was not detected, which correlates with the existing data about natural EVs since calnexin is expressed on the membrane of endoplasmic reticulum and Golgi apparatus [33].
Flow cytometry methods are most often used to investigate the molecular composition and size of cells. However, there are difficulties with the detection of results, since most flow cytometers are optimized for measuring biological particles in the cellular range from 2 to 30 microns [34]. Imaging flow cytometry has all the advantages of conventional flow cytometry, while also providing the additional value of being able to see the images of the cells/particles being analyzed. Using imaging flow cytometry, subtype analysis of EVs, profiling of circulating EVs for tissue-specific biomarker discovery, and functional analysis, as well as analysis of interaction of EVs with target cells, can be carried out [35].
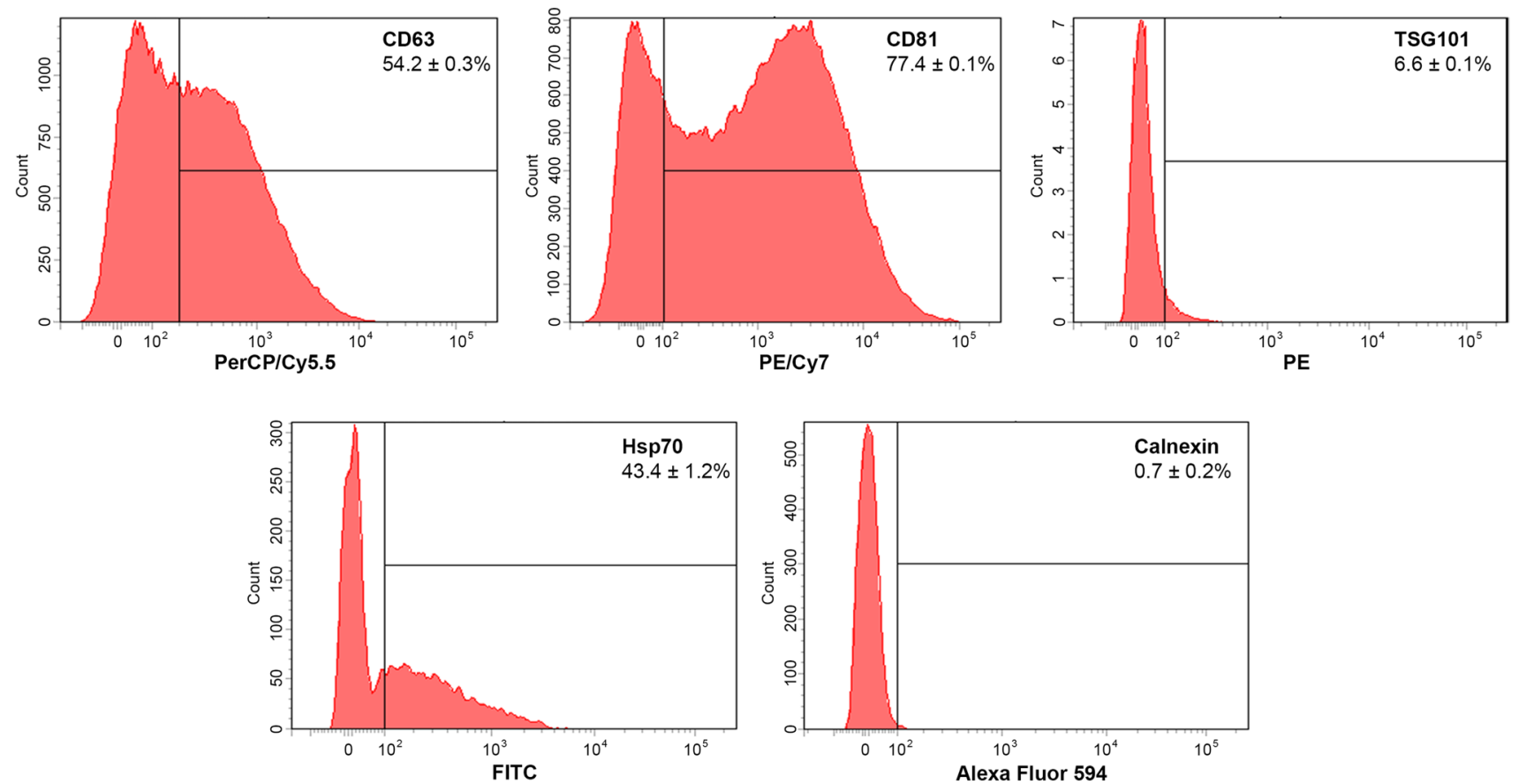

Fig. 3 Analysis of the expression of typical EV markers in the SH-SY5Y cell-derived CIMVs 

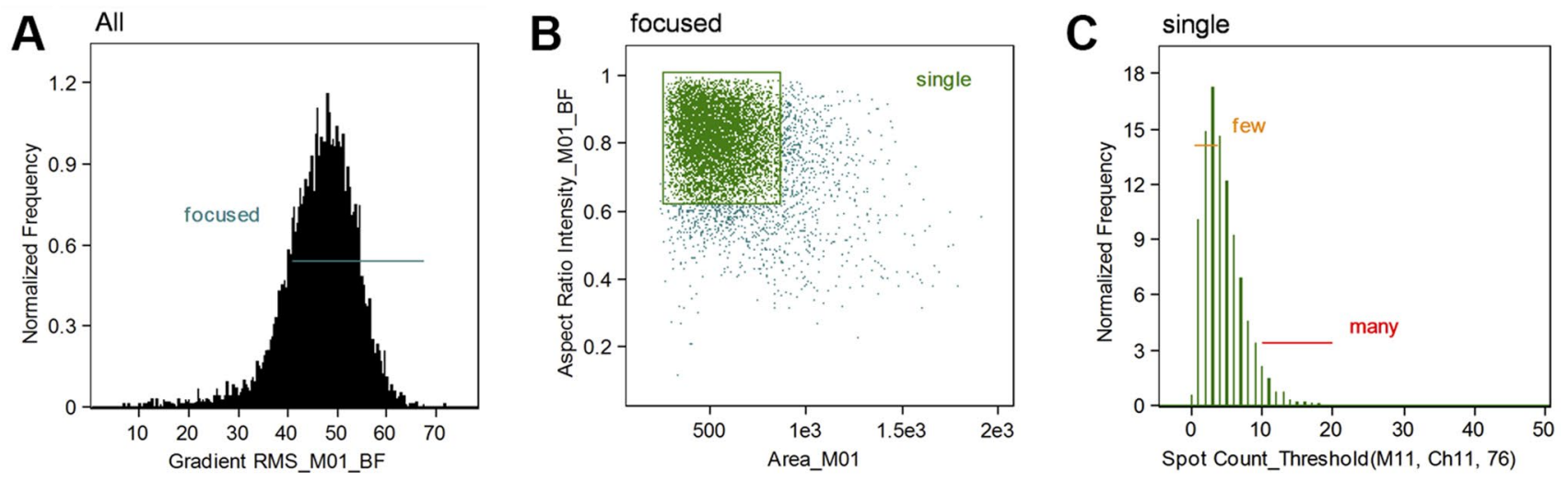

Spot Count_Threshold (M11, Ch11, 76)

\begin{tabular}{|l|l|l|}
\hline Population & Count & \%Gated \\
\hline single \& focused & 7178 & 100 \\
\hline many \& single \& focused & 436 & 6.07 \\
\hline few \& single \& focused & 3032 & 42.2 \\
\hline
\end{tabular}

D

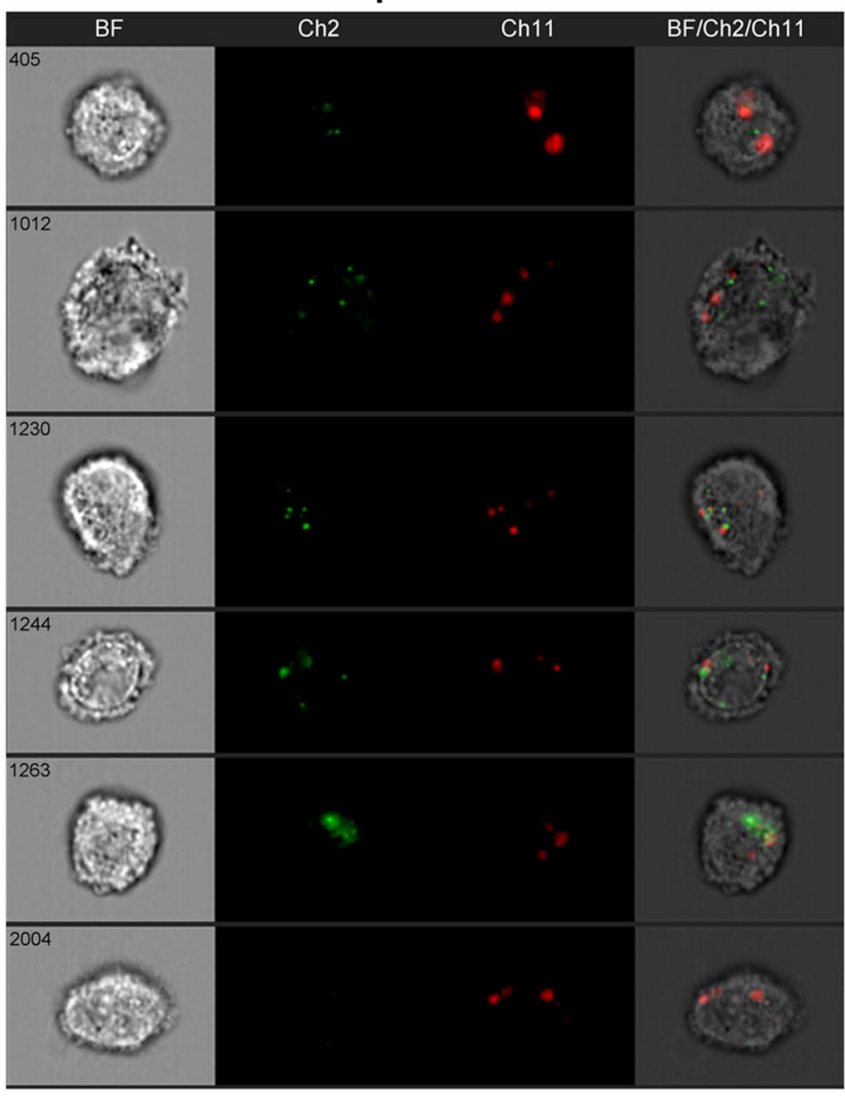

Fig. 4 Interaction of human bone marrow MSCs with SH-SY5Y cell-derived CIMVs analyzed using imaging flow cytometry. A, B, C Workflow diagrams show the IDEAS software algorithm. First, images of the MSCs in focus were selected (A), and then the relevant single cell suspension was selected (B), excluding multi-cell clusters and debris. The Spot Count algorithm was used to calculate the distribution of internalized CIMVs (C). D Two galleries of representa-

\section{many particles}

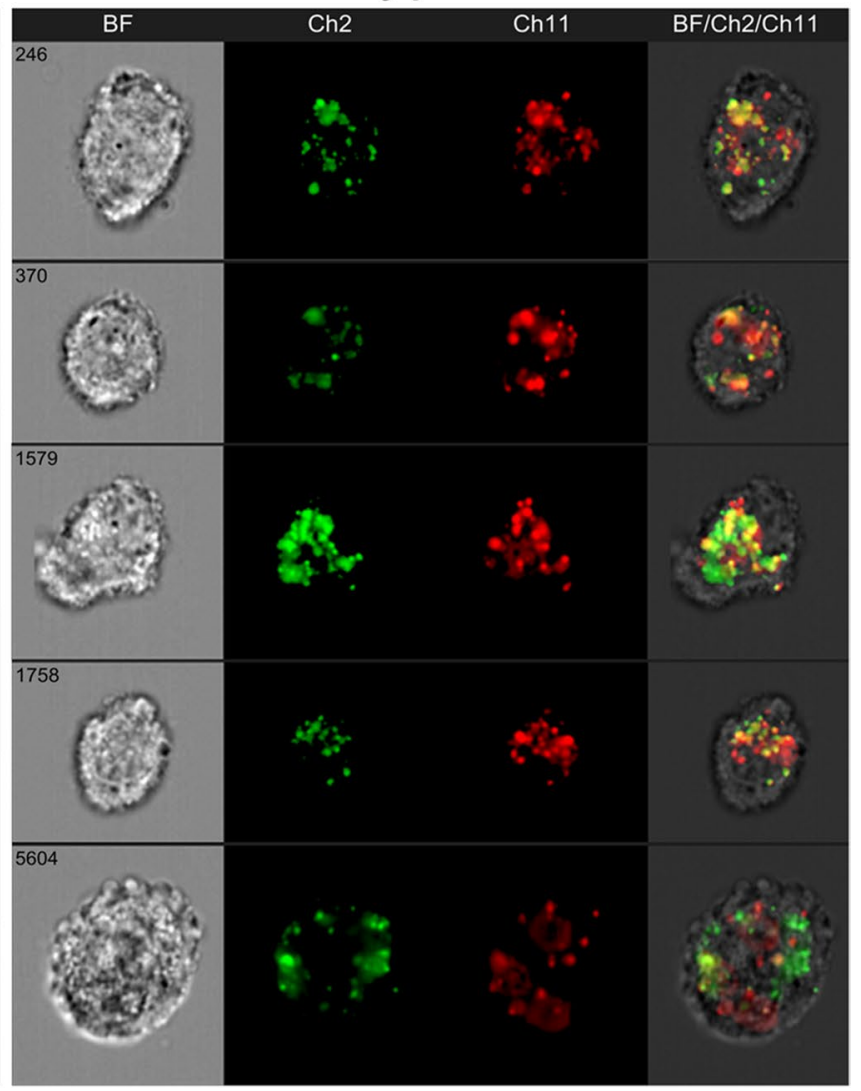

tive images of MSCs labeled with lipid DiO fluorescent dye after $24 \mathrm{~h}$ of incubation with CIMVs stained with DiD fluorescent dye and acquired with 5-laser ImageStreamX Mark II (Amnis-EMD-Millipore, USA) using $\times 60$ objective. Channels: BF, Brightfield (420$480 \mathrm{~nm})$; Ch2, FITC channel (480-560 nm); Ch11, Cy5/APC channel (642-745 nm); composite channel is overlay of BF, Ch2, and Ch11 
To analyze the interaction of CIMVs and MSCs, the cells and CIMVs were labelled with fluorescent dyes $\mathrm{DiO}$ (green spectrum, Ex/Em = 484/501 nm) and DiD (red spectrum, Ex/ $\mathrm{Em}=644 / 665 \mathrm{~nm}$ ), respectively, and co-cultured for $24 \mathrm{~h}$. After 24-h incubation, flow cytometry analysis showed that $48.27 \%$ of the analyzed MSCs contained CIMVs, of which $6.07 \%$ of MSCs in the single cell suspension contained a large number of CIMVs per cell, while $42.2 \%$ of MSCs contained a few CIMVs per cell (Fig. 4). Thus, a fusion of MSCs and SH-SY5Y cell-derived CIMVs was shown; it can lead to modulation of the MSC phenotype and functions in TME. It is known that tumor cell-derived EVs when interacting with MSCs can enhance their migration ability and expression of alpha-smooth muscle actin mRNA, as well as induce the release of (C-X-C motif) ligand 1 (CXCL-1), monocyte chemoattractant protein 1 (MCP-1), and interleukin 6 (IL-6) [36]. Increased secretion of IL-6 by mesenchymal stem cells may increase the secretion of endothelin-1 by tumor cells and induce activation of Akt and ERK in endothelial cells and enlarge their recruitment to TME and angiogenesis [37]. We have previously shown that SH-SY5Y cells can stimulate the formation of capillary-like structures by MSCs on a thin layer of Matrigel. [25]. It has also been shown that SH-SY5Y cell-derived CIMVs contain elevated levels of VEGF as compared to the parental cells, and stimulate angiogenesis in vitro and in vivo [38].

The influence of SH-SY5Y cell-derived CIMVs on the immunophenotype of MSCs was analyzed using flow cytometry. A dose-dependent decrease in the expression of the analyzed CD markers was noted in MSCs $24 \mathrm{~h}$ after the cultivation with SH-SY5Y cell-derived CIMVs in concentrations $100 \mu \mathrm{g} / \mathrm{ml}$ or $500 \mu \mathrm{g} / \mathrm{ml}$ (Fig. 5). Thus, the expression of CD29 in MSCs was reduced by $3.40 \%$ and by $14.79 \%$ after the addition of $100 \mu \mathrm{g} / \mathrm{ml}$ and $500 \mu \mathrm{g} / \mathrm{ml}$ SH-SY5Y-derived CIMVs, respectively. The expression of CD44 in MSCs was reduced by $3.77 \%$ and by $15.37 \%$ after SH-SY5Y-derived CIMVs in concentrations of $100 \mu \mathrm{g} / \mathrm{ml}$ and $500 \mu \mathrm{g} / \mathrm{ml}$, respectively. The expression of CD73 in MSCs was decreased by $3.95 \%$ and by $12.50 \%$ after SHSY5Y-derived CIMVs in concentrations of $100 \mu \mathrm{g} / \mathrm{ml}$ and $500 \mu \mathrm{g} / \mathrm{ml}$, respectively. The number of CD90-positve MSCs was decreased by $6.65 \%$ and by $18.63 \%$ after $100 \mu \mathrm{g} / \mathrm{ml}$ and $500 \mu \mathrm{g} / \mathrm{ml}$ of SH-SY5Y-derived CIMVs, respectively. The expression of CD105 in MSCs was reduced by $18.48 \%$ and by $37.60 \%$ after cultivation with SH-SY5Y cells in concentrations of $100 \mu \mathrm{g} / \mathrm{ml}$ and $500 \mu \mathrm{g} / \mathrm{ml}$, respectively (Fig. 4). CIMVs from SH-SY5Y tumor cells apparently alter the metabolism of stem cells. The loss of stem cell markers may indicate the beginning of the differentiation process in the MSCs. However, this assumption is for further investigation. The obtained data is consistent with studies conducted on prostate cancer cells, where an active exchange

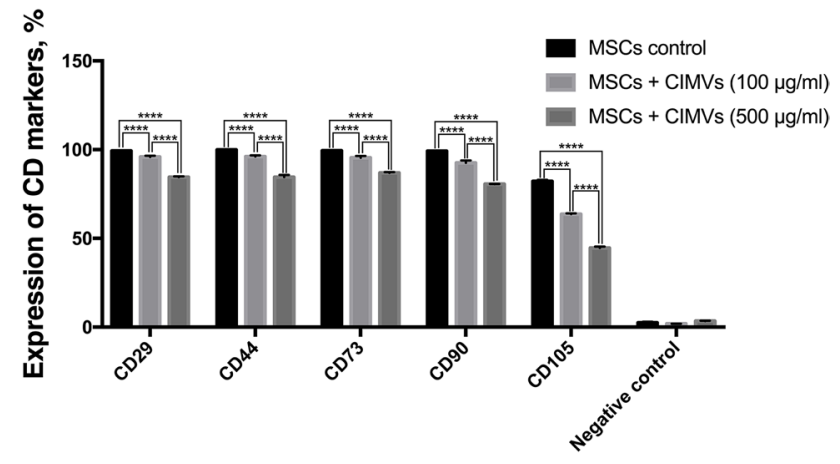

Fig. 5 Immunophenotyping of human bone marrow MSCs after the interaction with SH-SY5Y cell-derived CIMVs. Negative markers, hematopoietic cell markers: CD34, CD11b, CD19, CD45, and HLADR

of extracellular vesicles between stem and tumor cells in coculture was observed [39]. Such an exchange can play a dual role in cancer progression. On the one hand, the exchange of vesicles between stem and tumor cells leads to the development of drug resistance and enhances tumor aggression. On the other hand, these fundamental properties of cell communication can open up new therapeutic approaches to the delivery of drugs toward the tumor $[1,14,40,41]$.

\section{Conclusion}

Thus, we demonstrated internalization of SH-SY5Y cellderived CIMVs by MSCs, isolated from human bone marrow. Unlike natural EVs, cytochalasin B-induced membrane vesicles can be produced in large amount, which makes them a promising target for further research on EV-mediated intercellular communication in TME. However, further studies are required to determine the effect of SH-SY5Y cell-derived CIMVs on pro- or anti-oncogenic phenotypes and function of MSCs in TME.

Acknowledgements Some of the experiments were conducted using the equipment of the Interdisciplinary Center for Collective Use of Kazan Federal University, Interdisciplinary Center for Analytical Microscopy and Pharmaceutical Research and Education Center, Kazan (Volga Region) Federal University, Kazan, Russia. Imaging flow cytometry was carried out with support from Natasha S. Barteneva and Ivan A. Vorobjev from Nazarbayev University, Nur-Sultan, Republic of Kazakhstan.

Author Contribution KVK, isolation and immunophenotyping of MSCs; VVS, analysis of the interaction of SH-SY5Y cell-derived CIMVs using imaging flow cytometry; SSV, transmission electron microscopy; DSC and IYF, analysis of CD marker expression in the CIMVs; VVS, manuscript writing; DSC, manuscript editing; AAR, supervision and manuscript editing. 
Funding This work was funded by the Russian Science Foundation (RSF) grant 18-74-10044 and the subsidy allocated to KFU for the state assignment 0671-2020-0058 in the sphere of scientific activities and by the Kazan Federal University Strategic Academic Leadership Program (PRIORITY-2030). Russian Science Foundation,18-7410044 ,Valeriya V. Solovyeva

\section{Declarations}

Conflict of Interest The authors declare no competing interests.

\section{References}

1. Chulpanova, D. S., Kitaeva, K. V., James, V., Rizvanov, A. A., \& Solovyeva, V. V. (2018). Therapeutic prospects of extracellular vesicles in cancer treatment. Frontiers in Immunology, 9, 1534. https://doi.org/10.3389/fimmu.2018.01534

2. Lee, Y., El Andaloussi, S., \& Wood, M. J. (2012). Exosomes and microvesicles: Extracellular vesicles for genetic information transfer and gene therapy. Human Molecular Genetics, 21(R1), R125-134. https://doi.org/10.1093/hmg/dds317

3. O'Loghlen, A. (2018). Role for extracellular vesicles in the tumour microenvironment. Philosophical Transactions of the Royal Society of London. Series B, Biological Sciences, 373(1737), 20160488. https://doi.org/10.1098/rstb.2016.0488.

4. Lin, S. K., Moss, A. A., \& Riegelman, S. (1977). Iodipamide kinetics: Capacity-limited biliary excretion with simultaneous pseudo-first-order renal excretion. Journal of Pharmaceutical Sciences, 66(12), 1670-1674.

5. Kharaziha, P., Ceder, S., Li, Q., \& Panaretakis, T. (2012). Tumor cell-derived exosomes: A message in a bottle. Biochimica et Biophysica Acta, 1826(1), 103-111. https://doi.org/10.1016/j.bbcan. 2012.03.006

6. Zhang, J., Sun, D., Fu, Q., Cao, Q., Zhang, H., \& Zhang, K. (2016). Bone mesenchymal stem cells differentiate into myofibroblasts in the tumor microenvironment. Oncology Letters, 12(1), 644-650. https://doi.org/10.3892/ol.2016.4645

7. Mishra, P. J., Mishra, P. J., Humeniuk, R., Medina, D. J., Alexe, G., Mesirov, J. P., Ganesan, S., Glod, J. W., \& Banerjee, D. (2008). Carcinoma-associated fibroblast-like differentiation of human mesenchymal stem cells. Cancer Research, 68(11), 4331-4339. https://doi.org/10.1158/0008-5472.CAN-08-0943

8. Quante, M., Tu, S. P., Tomita, H., Gonda, T., Wang, S. S., Takashi, S., Baik, G. H., Shibata, W., Diprete, B., Betz, K. S., Friedman, R., Varro, A., Tycko, B., \& Wang, T. C. (2011). Bone marrowderived myofibroblasts contribute to the mesenchymal stem cell niche and promote tumor growth. Cancer Cell, 19(2), 257-272. https://doi.org/10.1016/j.ccr.2011.01.020

9. Lv, C., Dai, H., Sun, M., Zhao, H., Wu, K., Zhu, J., Wang, Y., Cao, X., Xia, Z., \& Xue, C. (2017). Mesenchymal stem cells induce epithelial mesenchymal transition in melanoma by paracrine secretion of transforming growth factor-beta. Melanoma Research, 27(2), 74-84. https://doi.org/10.1097/CMR.0000000000000325

10. Poggi, A., Varesano, S., \& Zocchi, M. R. (2018). How to hit mesenchymal stromal cells and make the tumor microenvironment immunostimulant rather than immunosuppressive. Frontiers in Immunology, 9, 262. https://doi.org/10.3389/fimmu.2018.00262

11. Poggi, A., \& Giuliani, M. (2016). Mesenchymal stromal cells can regulate the immune response in the tumor microenvironment. Vaccines, 4(4), 41. https://doi.org/10.3390/vaccines4040041

12. Khakoo, A. Y., Pati, S., Anderson, S. A., Reid, W., Elshal, M. F., Rovira, I. I., Nguyen, A. T., Malide, D., Combs, C. A., Hall,
G., Zhang, J., Raffeld, M., Rogers, T. B., Stetler-Stevenson, W., Frank, J. A., Reitz, M., \& Finkel, T. (2006). Human mesenchymal stem cells exert potent antitumorigenic effects in a model of Kaposi's sarcoma. The Journal of Experimental Medicine, 203(5), 1235-1247. https://doi.org/10.1084/jem.20051921

13. Qiao, L., Xu, Z., Zhao, T., Zhao, Z., Shi, M., Zhao, R. C., Ye, L., \& Zhang, X. (2008). Suppression of tumorigenesis by human mesenchymal stem cells in a hepatoma model. Cell Research, 18(4), 500-507. https://doi.org/10.1038/cr.2008.40

14. Chulpanova, D. S., Kitaeva, K. V., Tazetdinova, L. G., James, V., Rizvanov, A. A., \& Solovyeva, V. V. (2018). Application of mesenchymal stem cells for therapeutic agent delivery in anti-tumor treatment. Frontiers in Pharmacology, 9, 259. https://doi.org/10. 3389/fphar.2018.00259

15 Hill, B. S., Pelagalli, A., Passaro, N., \& Zannetti, A. (2017). Tumor-educated mesenchymal stem cells promote pro-metastatic phenotype. Oncotarget, 8(42), 73296-73311. https://doi.org/10. 18632/oncotarget.20265

16. Kidd, S., Spaeth, E., Dembinski, J. L., Dietrich, M., Watson, K., Klopp, A., Battula, V. L., Weil, M., Andreeff, M., \& Marini, F. C. (2009). Direct evidence of mesenchymal stem cell tropism for tumor and wounding microenvironments using in vivo bioluminescent imaging. Stem Cells, 27(10), 2614-2623. https://doi.org/ 10.1002/stem. 187

17. Klopp, A. H., Spaeth, E. L., Dembinski, J. L., Woodward, W. A., Munshi, A., Meyn, R. E., Cox, J. D., Andreeff, M., \& Marini, F. C. (2007). Tumor irradiation increases the recruitment of circulating mesenchymal stem cells into the tumor microenvironment. Cancer Research, 67(24), 11687-11695. https://doi.org/10.1158/ 0008-5472.CAN-07-1406

18. Chulpanova, D. S., Solovyeva, V. V., Kitaeva, K. V., Dunham, S. P., Khaiboullina, S. F., \& Rizvanov, A. A. (2018). Recombinant viruses for cancer therapy Biomedicines, 6(4), 94. https://doi.org/ 10.3390/biomedicines6040094

19. Gilazieva, Z. E., Tazetdinova, L. G., Arkhipova, S. S., Solovyeva, V. V., \& Rizvanov, A. A. (2016). Effect of cisplatin on ultrastructure and viability of adipose-derived mesenchymal stem cells. BioNanoScience, 6(4), 534-539. https://doi.org/10.1007/ s12668-016-0283-0

20. Gomzikova, M. O., Aimaletdinov, A. M., Bondar, O. V., Starostina, I. G., Gorshkova, N. V., Neustroeva, O. A., Kletukhina, S. K., Kurbangaleeva, S. V., Vorobev, V. V., Garanina, E. E., Persson, J. L., Jeyapalan, J., Mongan, N. P., Khaiboullina, S. F., \& Rizvanov, A. A. (2020). Immunosuppressive properties of cytochalasin B-induced membrane vesicles of mesenchymal stem cells: Comparing with extracellular vesicles derived from mesenchymal stem cells. Scientific Reports, 10, 10740. https://doi.org/10.1038/ S41598-020-67563-9

21. Kitaeva, K. V., Prudnikov, T. S., Gomzikova, M. O., Kletukhina, S. K., James, V., Rizvanov, A. A., \& Solovyeva, V. V. (2019). Analysis of the interaction and proliferative activity of adenocarcinoma, peripheral blood mononuclear and mesenchymal stromal cells after co-cultivation in vitro. BioNanoScience, 9(2), 502-509. https://doi.org/10.1007/s12668-019-00625-z

22. Solovyeva, V. V., Salafutdinov, I. I., Tazetdinova, L. G., Khaiboullina, S. F., Masgutov, R. F., \& Rizvanov, A. A. (2014). Genetic modification of adipose derived stem cells with recombinant plasmid DNA pBud-VEGF-FGF2 results in increased of IL-8 and MCP-1 secretion. Journal of Pure and Applied Microbiology, 8(Spl. Edn. 2), 523-528.

23. Solovyeva, V. V., Kolobynina, K. G., Gomzikova, M. O., Tazetdinova, L. G., Zhuravleva, M. N., Slepak, V. Z., \& Rizvanov, A. A. (2015). Effect of tescalcin overexpression on osteogenic differentiation of human mesenchymal stem cells. Genes \& Cells, 10(4), 90-93.

24. Gomzikova, M., Kletukhina, S., Kurbangaleeva, S., \& Rizvanov, A. (2018). Evaluation of cytochalasin B-induced membrane vesicles 
fusion specificity with target cells. BioMed Research International, 2018, 7053623. https://doi.org/10.1155/2018/7053623

25. Rizvanov, A. A., Yalvac, M. E., Shafigullina, A. K., Salafutdinov, I. I., Blatt, N. L., Sahin, F., Kiyasov, A. P., \& Palotas, A. (2010). Interaction and self-organization of human mesenchymal stem cells and neuro-blastoma SH-SY5Y cells under co-culture conditions: A novel system for modeling cancer cell micro-environment. European Journal of Pharmaceutics and Biopharmaceutics, 76(2), 253-259. https://doi.org/10.1016/j.ejpb.2010.05.012

26. Szatanek, R., Baran, J., Siedlar, M., \& Baj-Krzyworzeka, M. (2015). Isolation of extracellular vesicles: Determining the correct approach (Review). International Journal of Molecular Medicine, 36(1), 11-17. https://doi.org/10.3892/ijmm.2015.2194

27. Wu, K., Xing, F., Wu, S. Y., \& Watabe, K. (2017). Extracellular vesicles as emerging targets in cancer: Recent development from bench to bedside. Biochimica et Biophysica Acta - Reviews on Cancer, 1868(2), 538-563. https://doi.org/10.1016/j.bbcan.2017. 10.001

28. Chang, C. F., \& Li, C. Z. (1986). Experimental studies on the mechanism of anti-platelet aggregation action of motherwort. Zhong Xi Yi Jie He Za Zhi, 6(1), 39-40.

29. Blavier, L., Yang, R. M., \& DeClerck, Y. A. (2020). The tumor microenvironment in neuroblastoma: New players, new mechanisms of interaction and new perspectives. Cancers (Basel), 12(10), 2912. https://doi.org/10.3390/cancers12102912

30. Pick, H., Schmid, E. L., Tairi, A. P., Ilegems, E., Hovius, R., \& Vogel, H. (2005). Investigating cellular signaling reactions in single attoliter vesicles. Journal of the American Chemical Society, 127(9), 2908-2912. https://doi.org/10.1021/ja044605x

31. Prada, I., \& Meldolesi, J. (2016). Binding and fusion of extracellular vesicles to the plasma membrane of their cell targets. International Journal of Molecular Sciences, 17(8), 1296. https://doi. org/10.3390/ijms17081296

32. Campos-Silva, C., Suárez, H., Jara-Acevedo, R., Linares-Espinós, E., Martinez-Piñeiro, L., Yáñez-Mó, M., \& Valés-Gómez, M. (2019). High sensitivity detection of extracellular vesicles immune-captured from urine by conventional flow cytometry. Scientific Reports, 9(1), 2042. https://doi.org/10.1038/ s41598-019-38516-8

33. Thery, C., et al. (2018). Minimal information for studies of extracellular vesicles 2018 (MISEV2018): A position statement of the International Society for Extracellular Vesicles and update of the MISEV2014 guidelines. Journal of Extracellular Vesicles, 7(1), 1535750. https://doi.org/10.1080/20013078.2018.1535750

34. Lannigan, J., \& Erdbruegger, U. (2017). Imaging flow cytometry for the characterization of extracellular vesicles. Methods, 112, 55-67. https://doi.org/10.1016/j.ymeth.2016.09.018
35. Mastoridis, S., Bertolino, G. M., Whitehouse, G., Dazzi, F., Sanchez-Fueyo, A., \& Martinez-Llordella, M. (2018). Multiparametric analysis of circulating exosomes and other small extracellular vesicles by advanced imaging flow cytometry. Frontiers in Immunology, 9, 1583. https://doi.org/10.3389/fimmu.2018.01583

36. Haga, H., Yan, I. K., Takahashi, K., Wood, J., Zubair, A., \& Patel, T. (2015). Tumour cell-derived extracellular vesicles interact with mesenchymal stem cells to modulate the microenvironment and enhance cholangiocarcinoma growth. Journal of Extracellular Vesicles, 4, 24900. https://doi.org/10.3402/jev.v4.24900

37. Huang, W. H., Chang, M. C., Tsai, K. S., Hung, M. C., Chen, H. L., \& Hung, S. C. (2013). Mesenchymal stem cells promote growth and angiogenesis of tumors in mice. Oncogene, 32(37), 4343-4354. https://doi.org/10.1038/onc.2012.458

38. Gomzikova, M. O., Zhuravleva, M. N., Miftakhova, R. R., Arkhipova, S. S., Evtugin, V. G., Khaiboullina, S. F., Kiyasov, A. P., Persson, J. L., Mongan, N. P., Pestell, R. G., \& Rizvanov, A. A. (2017). Cytochalasin B-induced membrane vesicles convey angiogenic activity of parental cells. Oncotarget, 8(41), 70496-70507. https://doi.org/10.18632/oncotarget.19723

39. Probert, C., Dottorini, T., Speakman, A., Hunt, S., Nafee, T., Fazeli, A., Wood, S., Brown, J. E., \& James, V. (2019). Communication of prostate cancer cells with bone cells via extracellular vesicle RNA. A potential mechanism of metastasis. Oncogene, 38(10), 1751-1763. https://doi.org/10.1038/s41388-018-0540-5

40. Chulpanova, D. S., Gilazieva, Z. E., Kletukhina, S. K., Aimaletdinov, A. M., Garanina, E. E., James, V., Rizvanov, A. A., \& Solovyeva, V. V. (2021). Cytochalasin B-induced membrane vesicles from human mesenchymal stem cells overexpressing IL2 are able to stimulate CD8+ T-killers to kill human triple negative breast cancer cells. Biology (Basel), 10(2), 141. https://doi.org/10.3390/biology10020141

41. Chulpanova, D. S., Solovyeva, V. V., James, V., Arkhipova, S. S., Gomzikova, M. O., Garanina, E. E., Akhmetzyanova, E. R., Tazetdinova, L. G., Khaiboullina, S. F., \& Rizvanov, A. A. (2020). Human mesenchymal stem cells overexpressing interleukin 2 can suppress proliferation of neuroblastoma cells in co-culture and activate mononuclear cells in vitro. Bioengineering (Basel), 7(2), 59. https://doi.org/10.3390/bioengineering7020059

Publisher's Note Springer Nature remains neutral with regard to jurisdictional claims in published maps and institutional affiliations. 